PROCEEDINGS OF THE

AMERICAN MATHEMATICAL SOCIETY

Volume 131, Number 8, Pages 2359-2369

S 0002-9939(03)06993-4

Article electronically published on March 11, 2003

\title{
NONLINEAR HEMIVARIATIONAL INEQUALITIES OF SECOND ORDER USING THE METHOD OF UPPER-LOWER SOLUTIONS
}

\author{
NIKOLAOS C. KOUROGENIS AND NIKOLAOS S. PAPAGEORGIOU
}

(Communicated by David S. Tartakoff)

\begin{abstract}
In this paper we examine a nonlinear hemivariational inequality of second order. The differential operator is set-valued, nonlinear and depends on both $x$ and its gradient $D x$. The same is true for the zero order term $f$, while the right-hand side nonlinearity satisfies a one-sided Lipschitz condition. We use the method of upper and lower solutions, coupled with truncation and penalization techniques and the fixed point theory for multifunctions in an ordered Banach space.
\end{abstract}

\section{INTRODUCTION}

The method of upper and lower solutions offers a powerful tool to establish the existence of solutions and multiple solutions for initial and boundary value problems. This method generates solutions of the problem located in the order interval with the upper and lower solutions serving as bounds.

In this paper we employ this method to study a nonlinear elliptic differential equation of second order involving the subdifferential term of a locally Lipschitz potential which can be nonsmooth. Such equations are known in the literature as "hemivariational inequalities" and have applications in mechanics and engineering (see the books of Naniewicz-Panagiotopoulos [12] and Panagiotopoulos [13). Hemivariational inequalities also incorporate as special case problems with discontinuities. This is the case when the nonsmooth potential is the indefinite integral of a bounded function. Such problems were studied by Ambrosetti-Badiale 1], CarlDietrich [2], Chang [3], Kourogenis-Papageorgiou [9] and Stuart [16], using a variety of methods. The method of upper and lower solutions was employed in the context of elliptic equations by Deuel-Hess [4, Carl-Dietrich 2, V. K. Le [10, Stuart [16] and in the book of Heikkila-Lakshmikantham [6], where the interested reader can find a detailed bibliography on the subject. Finally we should also mention the recent interesting work of V. K. Le [11, who extended the method to elliptic variational inequalities (see also Papageorgiou-Papalini-Vercillo [14]).

Received by the editors October 9, 2001.

2000 Mathematics Subject Classification. Primary 35J50, 35J85, 35R70.

Key words and phrases. Upper solution, lower solution, upper semicontinuous multifunction, lower semicontinuous multifunction, graph measurability, operator of type $S_{+}$, pseudomonotone operator, truncation map, penalty map, coercive map, order interval.

The first author was supported by a grant of the National Scholarship Foundation of Greece (I.K.Y.). 
In addition to the presence of the nonmonotone subdifferential term (nonconvex potential), another novel feature of our problem is the multivalued nonlinear differential operator and the nonlinear weakly monotone right hand side. These facts in general cannot guarantee uniqueness and so we cannot use the classical fixed point theorems and we need to appeal to a recent result of Heikkila-Hu [5].

\section{MAThematicAl BACKGROUND}

Let $(\Omega, \Sigma, \mu)$ be a finite measure space and $X$ a separable Banach space. We introduce the following hyperspaces: $P_{f(c)}(X)=\{A \subseteq X$ : nonempty, closed (and convex) $\}$ and $P_{(w) k(c)}(X)=\{A \subseteq X$ : nonempty, (weakly) compact (and convex) $\}$. A multifunction $F: \Omega \rightarrow 2^{X} \backslash\{\emptyset\}$ is said to be "graph measurable", if $G r F=$ $\{(\omega, x) \in \Omega \times X: x \in F(\omega)\} \in \Sigma \times B(X)$, with $B(X)$ being the Borel $\sigma$-field of $X$. A multifunction $F: \Omega \rightarrow P_{f}(X)$ is said to be "measurable", if for all $x \in X, \omega \rightarrow d(x, F(\omega))=\inf [\|x-y\|: y \in F(\omega)]$ is measurable. For $P_{f}(X)$-valued multifunctions, measurability implies graph measurability and the converse is true if $\Sigma$ is $\mu$-complete. For $1 \leq p \leq \infty$, by $S_{F}^{p}$ we denote the set $L^{p}(\Omega, X)$-selectors of $F$, i.e. $S_{F}^{p}=\left\{f \in L^{p}(\Omega, X): f(\omega) \in F(\omega) \mu-\right.$ a.e. $\}$.

Let $V, Y$ be Hausdorff topological spaces and $F: V \rightarrow 2^{Y} \backslash\{\emptyset\}$. We say that $F$ is lower semicontinuous (lsc for short) (resp. upper semicontinuous (usc for short)), if for all $C \subseteq Y$ closed, the set $F^{+}(C)=\{v \in V: F(v) \subseteq C\}$ (resp. $\left.F^{-}(C)=\{v \in V: F(v) \cap C \neq \emptyset\}\right)$ is closed. A $P_{f}(Y)$-valued, usc multifunction has closed graph. The converse is true if $F$ is locally compact, i.e. for all $v \in V$ there exists a neighborhood $U$ of $v$ such that $\overline{F(U)} \in P_{k}(Y)$. If $Y$ is a metric space and $A, C \subseteq Y$ nonempty sets, we define $h^{*}(A, C)=\sup [d(a, C): a \in A]$ (the excess of $A$ over $C$ ) and $h(A, C)=\max \left\{h^{*}(A, C), h^{*}(C, A)\right\}$ (the Hausdorff distance between $A$ and $C$ ). On $P_{f}(Y), h$ is a metric called a "Hausdorff metric" and if $Y$ is complete, so is $\left(P_{f}(Y), h\right)$. For more about multifunctions we refer to Hu-Papageorgiou [7].

Suppose $X$ is a reflexive Banach space, $X^{*}$ its topological dual and $A: X \rightarrow$ $P_{w k c}\left(X^{*}\right)$ a bounded map (i.e. maps bounded sets to bounded sets). We say that $A$ is of type $S_{+}$if whenever $x_{n} \stackrel{w}{\rightarrow} x$ in $X, x_{n}^{*} \stackrel{w}{\rightarrow} x^{*}$ in $X^{*}, x_{n}^{*} \in A\left(x_{n}\right), n \geq 1$, and $\lim \sup \left\langle x_{n}^{*}, x_{n}-x\right\rangle \leq 0$ (here by $\langle\cdot, \cdot\rangle$ we denote the duality brackets for $X$ and $\left.X^{*}\right)$, we have $x^{*} \in A(x)$ and $x_{n} \rightarrow x$ in $X$. Any such $A$ is also pseudomonotone. For details see Hu-Papageorgiou 7] and Showalter [15].

Finally for $X$ a Banach space and $\phi: X \rightarrow \mathbb{R}$ a locally Lipschitz function, we define the generalized directional derivative of $\phi$ at $x \in X$ by

$$
\phi^{0}(x ; h)=\lim _{\substack{x^{\prime} \rightarrow x \\ \lambda \downarrow 0}} \frac{\phi\left(x^{\prime}+\lambda h\right)-\phi\left(x^{\prime}\right)}{\lambda} .
$$

The function $h \rightarrow \phi^{0}(x ; h)$ is continuous sublinear and so by the Hahn-Banach theorem it is the support function of a nonempty $w$-compact and convex set $\partial \phi(x)=$ $\left\{x^{*} \in X^{*}:\left(x^{*}, h\right) \leq \phi^{0}(x ; h)\right.$ for all $\left.h \in X\right\}$. The set $\partial \phi(x)$ is known as the (Clarke) subdifferential of $\phi$ at $x$ (see Chang [3] or Hu-Papageorgiou [8]). The multifunction $x \rightarrow \partial \phi(x)$ is usc from $X$ into $X^{*}$ furnished with the $w^{*}$-topology. If $\phi \in C^{1}(X)$, then $\partial \phi(x)=\left\{\phi^{\prime}(x)\right\}$. 


\section{Auxiliary Results}

Let $Z \subseteq \mathbb{R}^{N}$ be a bounded domain with $C^{1}$-boundary $\Gamma$. We examine the following problem:

$$
\left\{\begin{array}{l}
-\operatorname{diva}(z, x(z), D x(z))+f(z, x(z), D x(z))+\partial j(z, x(z)) \ni \theta(x(z)) \\
x_{\left.\right|_{\Gamma}}=0 .
\end{array}\right.
$$

Here $a: Z \times \mathbb{R} \times \mathbb{R}^{N} \rightarrow 2^{\mathbb{R}^{N}} \backslash\{\emptyset\}$ and for any $x \in W_{0}^{1, p}(Z)$ by diva $(z, x(z), D x(z))$ we understand the following set:

$$
\left\{\operatorname{divv}(z): v \in L^{q}\left(Z, \mathbb{R}^{N}\right), v(z) \in a(z, x(z), D x(z)) \text { a.e. on } Z\right\}
$$

with $\frac{1}{p}+\frac{1}{q}=1$ (i.e. $v \in S_{a(\cdot, x(\cdot), D x(\cdot))}^{q}$ ). By a solution of (1), we mean a function $x \in W_{0}^{1, p}(Z)$ for which there exist $v \in S_{a(\cdot, x(\cdot), D x(\cdot))}^{q}$ and $u^{*} \in S_{\partial j(\cdot, x(\cdot))}^{q}$ such that $-\operatorname{divv}(z)+f(z, x(z), D x(z))+u^{*}(z)=\theta(x(z))$ a.e. on $Z$. Our hypotheses on the data of (1) are:

$\mathbf{H}(a): a: Z \times \mathbb{R} \times \mathbb{R}^{N} \rightarrow P_{k c}\left(\mathbb{R}^{N}\right)$ is a multifunction such that

(i) $(z, x, \xi) \rightarrow a(z, x, \xi)$ is graph measurable;

(ii) for almost all $z \in Z$ and all $x \in \mathbb{R}, \xi \rightarrow a(z, x, \xi)$ is strictly monotone;

(iii) for almost all $z \in Z,(x, \xi) \rightarrow a(z, x, \xi)$ has a closed graph and for almost all $z \in Z$ and all $\xi \in \mathbb{R}^{N}, x \rightarrow a(z, x, \xi)$ is lsc;

(iv) for almost all $z \in Z$, all $x \in \mathbb{R}$, all $\xi \in \mathbb{R}^{N}$ and all $v \in a(z, x, \xi),\|v\| \leq$ $b_{1}(z)+c_{1}\left(|x|^{p-1}+\|\xi\|^{p-1}\right)$ with $b_{1} \in L^{q}(Z), c_{1}>0 ;$

(v) for almost all $z \in Z$, all $x \in \mathbb{R}$, all $\xi \in \mathbb{R}^{N}$ and all $v \in a(z, x, \xi),(v, \xi)_{\mathbb{R}^{N}}$ $\geq \gamma_{1}\|\xi\|^{p}-\gamma_{2}(z), \gamma_{1}>0, \gamma_{2} \in L^{1}(Z)_{+}$.

Remark. The multifunction $a(z, x, \xi)$ can be of the form $a(z, x, \xi)=a_{0}(z, x) \partial k(z, \xi)$, with $a_{0}$ a Caratheodory function on $Z \times \mathbb{R}$ into $\mathbb{R}_{+}$and $k: Z \times \mathbb{R}^{N} \rightarrow \mathbb{R}$ a Caratheodory strictly convex integrand which is not necessarily differentiable in the $\xi \in \mathbb{R}^{N}$ variable. If $k(z, \xi)=\frac{1}{p}\|\xi\|^{p}$, then $a(z, x, \xi)=a_{0}(z, x)\|\xi\|^{p-2} \xi$ which is a generalization of the $p$-Laplacian. Recently, problems involving the $p$-Laplacian have been the object of intense research.

Before presenting our conditions on $f, j$ and $\theta$, we need to define what we mean by an upper and a lower solution for problem (1). Let $V: W_{0}^{1, p}(Z) \rightarrow P_{w k c}\left(W^{-1, q}(Z)\right)$ be defined by $V(x)=\left\{-\right.$ divv $\left.: v \in S_{a(\cdot, x(\cdot), D x(\cdot))}^{q}\right\}$. By $\langle\cdot, \cdot \cdot\rangle$ we denote the duality brackets for the dual pair $W_{0}^{1, p}(Z)$ and $W^{-1, q}(Z)$.

Definition. (a) A function $\phi \in W^{1, p}(Z), \phi_{\left.\right|_{\Gamma}} \geq 0$ is an "upper solution" for problem (1) if there exist $v_{+}^{*} \in V(\phi)$ and $u_{+} \in S_{\partial j(\cdot, \phi(\cdot))}^{r^{\prime}}, \quad\left(\frac{1}{r}+\frac{1}{r^{\prime}}=1,1 \leq r<p^{*}=\right.$ $\left\{\begin{array}{ll}\frac{N p}{N-p} & \text { if } N>p, \\ +\infty & \text { otherwise }\end{array}\right)$ such that for all $y \in W_{0}^{1, p}(Z)_{+}$,

$$
\left\langle v_{+}^{*}, y\right\rangle+\int_{Z} f(z, \phi, D \phi) y d z+\int_{Z} u_{+} y d z \geq \int_{Z} \theta(\phi) y d z
$$

$\left(y \in W_{0}^{1, p}(Z)_{+}\right.$if $y \in W_{0}^{1, p}(Z)$ and $y(z) \geq 0$ a.e. $)$. 
(b) A function $\psi \in W^{1, p}(Z), \phi_{\left.\right|_{\Gamma}} \leq 0$ is a "lower solution" for problem (1) if there exist $v_{-}^{*} \in V(\psi)$ and $u_{-} \in S_{\partial j(\cdot, \psi(\cdot))}^{r^{\prime}}$ such that for all $y \in W_{0}^{1, p}(Z)_{+}$

$$
\left\langle v_{-}^{*}, y\right\rangle+\int_{Z} f(z, \psi, D \psi) y d z+\int_{Z} u_{-} y d z \leq \int_{Z} \theta(\psi) y d z .
$$

$\mathbf{H}_{0}$ : There exist an upper solution $\phi$ and a lower solution $\psi$ for problem (1) such that $\psi(z) \leq \phi(z)$ a.e. on $Z$.

$\mathbf{H}(j): j: Z \times \mathbb{R} \rightarrow \mathbb{R}$ is a function such that

(i) for all $x \in \mathbb{R}, z \rightarrow j(z, x)$ is measurable;

(ii) for almost all $z \in Z, x \rightarrow j(z, x)$ is locally Lipschitz;

(iii) for almost all $z \in Z$, all $x \in[\psi(z), \phi(z)]$ and all $u^{*} \in \partial j(z, x),\left|u^{*}\right|$ $\leq b_{2}(z)$ with $b_{2} \in L^{r^{\prime}}(Z)$.

$\mathbf{H}(f): f: Z \times \mathbb{R} \times \mathbb{R}^{N} \rightarrow \mathbb{R}$ is a function such that

(i) for all $(x, \xi) \in \mathbb{R} \times \mathbb{R}^{N}, z \rightarrow f(z, x, \xi)$ is measurable;

(ii) for almost all $z \in Z,(x, \xi) \rightarrow f(z, x, \xi)$ is continuous;

(iii) for almost all $z \in Z$, all $x \in[\psi(z), \phi(z)]$ and all $\xi \in \mathbb{R}^{N}$ we have $|f(z, x, \xi)| \leq b_{3}(z)+c_{3}\|\xi\|^{p-1}$ with $b_{3} \in L^{q}(Z), c_{3}>0$.

$\mathbf{H}(\theta): \theta: \mathbb{R} \rightarrow \mathbb{R}$ is a function such that for some $M>0, x \rightarrow \theta(x)+M x$ is nondecreasing and $\theta(\phi(\cdot)), \theta(\psi(\cdot)) \in L^{q}(Z)$.

We introduce the truncation map $\tau: W^{1, p}(Z) \rightarrow W^{1, p}(Z)$ defined by

$$
\tau(x)= \begin{cases}\phi(z) & \text { if } \phi(z)<x(z) \\ x(z) & \text { if } \psi(z) \leq x(z) \leq \phi(z) \\ \psi(z) & \text { if } x(z)<\psi(z)\end{cases}
$$

It is easy to see that $\tau$ is continuous. Also we shall use the penalty function $\beta: Z \times \mathbb{R} \rightarrow \mathbb{R}$ defined by

$$
\beta(z, x)= \begin{cases}|x|^{p-2} x-|\phi(z)|^{p-2} \phi(z) & \text { if } \phi(z)<x, \\ 0 & \text { if } \psi(z) \leq x \leq \phi(z), \\ |x|^{p-2} x-|\psi(z)|^{p-2} \psi(z) & \text { if } x<\psi(z) .\end{cases}
$$

Clearly $\beta$ is a Caratheodory function. For almost all $z \in Z$ and all $x \in \mathbb{R}$ we have $|\beta(z, x)| \leq b_{4}(z)+c_{4}|x|^{p-1}$ with $b_{4} \in L^{q}(Z), c_{4}>0$, and for all $x \in L^{p}(Z)$,

$$
\int_{Z} \beta(z, x(z)) x(z) d z \geq c_{5}\|x\|_{p}^{p}-c_{6}, \quad c_{5}, c_{6}>0 .
$$

Moreover, let $Q: Z \times \mathbb{R} \rightarrow P_{f c}(\mathbb{R})$ be defined by

$$
Q(z, x)= \begin{cases}{\left[u_{+}(z),+\infty\right)} & \text { if } \phi(z)<x \\ \mathbb{R} & \text { if } \psi(z) \leq x \leq \phi(z) \\ \left(-\infty, u_{-}(z)\right] & \text { if } x<\psi(z)\end{cases}
$$

and for $x \in W_{0}^{1, p}(Z)$ set $E(z, x(z))=\partial j(z, \tau(x)(z)) \cap Q(z, x(z))$. In what follows $K=[\psi, \phi]=\left\{x \in W^{1, p}(Z): \psi(z) \leq x(z) \leq \phi(z)\right.$ a.e. on $\left.Z\right\}$. Given $w \in K$ we consider the following auxiliary problem:

$$
\left\{\begin{array}{l}
-\operatorname{diva}(z, \tau(x)(z), D x(z))+f(z, \tau(x)(z), D \tau(x)(z))+M \tau(x)(z) \\
+\rho \beta(z, x(z))+E(z, x(z)) \ni \theta(w(z))+M w(z) \text { a.e. on } Z, \\
x_{\left.\right|_{\Gamma}}=0, \rho>0 .
\end{array}\right\}
$$


First we solve (2). To this end let $V_{1}: W_{0}^{1, p}(Z) \rightarrow P_{w k c}\left(W^{-1, q}(Z)\right)$ be defined by

$$
V_{1}(x)=\left\{-d i v v: v \in S_{a(\cdot, \tau(x)(\cdot), D x(\cdot))}^{q} .\right.
$$

Also for fixed $x \in W_{0}^{1, p}(Z)$ we consider the operator $L_{x}: W_{0}^{1, p}(Z) \rightarrow 2^{W^{-1, q}(Z)}$ defined by $L_{x}(y)=\left\{-\operatorname{divu}: u \in S_{a(\cdot, \tau(x)(\cdot), D y(\cdot))}^{q}\right\}$.

Lemma 1. If hypotheses $\mathrm{H}(a)$ hold, then for every $x \in W_{0}^{1, p}(Z), y \rightarrow L_{x}(y)$ is maximal monotone.

Proof. By virtue of hypotheses $\mathrm{H}(a)(\mathrm{i})$ and (iv), $S_{a(\cdot, \tau(x)(\cdot), D y(\cdot))}^{q} \neq \emptyset$, and it is weakly compact and convex. Moreover, because of hypothesis $\mathrm{H}(a)(\mathrm{ii}), L_{x}$ is clearly monotone. So in order to check the maximality of $L_{x}$ it suffices to show that for every $y, h \in W_{0}^{1, p}(Z), t \rightarrow L(y+t h)$ is usc from $[0,1]$ into $W^{-1, q}(Z)_{w}$ (see HuPapageorgiou [7], p. 309). Evidently $L_{x}$ is locally compact and so it is enough to show that $t \rightarrow L_{x}(y+t h)$ has a graph which is sequentially closed in $[0,1] \times$ $W^{-1, q}(Z)_{w}$ (recall that on bounded sets of $W^{-1, q}(Z)$ the relative weak topology is metrizable). So let $t_{n} \rightarrow t$ in $[0,1], v_{n}^{*} \rightarrow v^{*}$ in $W^{-1, q}(Z)$ and $v_{n}^{*} \in L_{x}(y+t h), n \geq$ 1. We have $v_{n}^{*}=-\operatorname{divu}_{n}$ with $u_{n} \in S_{a\left(\cdot, \tau(x)(\cdot), D\left(y+t_{n} y\right)(\cdot)\right)}^{q}, n \geq 1$. Because of hypotheses $\mathrm{H}(a)$ (iv), we have that $\left\{u_{n}\right\}_{n \geq 1} \subseteq L^{q}\left(Z, \mathbb{R}^{N}\right)$ is bounded. So we may assume that $u_{n} \stackrel{w}{\rightarrow} u$ in $L^{q}\left(Z, \mathbb{R}^{N}\right)$. Using proposition VII.3.9, p. 694 , of HuPapageorgiou [7], we have

$$
u(z) \in \text { conv } \lim \sup a\left(z, \tau(x)(z), D\left(y+t_{n} h\right)(z)\right) \subseteq a(z, \tau(x)(z), D(y+t h)(z))
$$

a.e. on $Z$, where the last inclusion follows from the fact that $a$ has closed convex values and for almost all $z \in Z, a(z, \cdot, \cdot)$ has closed graph (see $\mathrm{H}(a)$ (iii)). Note that - divu $_{n} \stackrel{w}{\rightarrow}-$ divu in $W^{-1, q}(Z)$ and so $v_{n}^{*} \stackrel{w}{\rightarrow} v^{*}=-$ divu in $W^{-1, q}(Z)$, with $u \in S_{a(\cdot, \tau(x)(\cdot), D(y+t h)(\cdot))}^{q}$, i.e. $v^{*} \in L_{x}(\cdot)$ is maximal monotone.

Proposition 2. If hypotheses $\mathrm{H}(a)$ hold, then $V_{1}$ is a multivalued operator of type $S_{+}$.

Proof. Let $\left\{x_{n}\right\}_{n \geq 1} \subseteq W_{0}^{1, p}(Z)$ and $v_{n}^{*} \in V_{1}\left(x_{n}\right), n \geq 1$, be two sequences such that

$$
x_{n} \stackrel{w}{\rightarrow} x \text { in } W_{0}^{1, p}(Z), v_{n}^{*} \stackrel{w}{\rightarrow} v^{*} \text { in } W^{-1, q}(Z) \text { and } \limsup _{n \rightarrow \infty}\left\langle v_{n}^{*}, x_{n}-x\right\rangle \leq 0 .
$$

We have that $v_{n}^{*}=-\operatorname{div}_{n}$ with $v_{n} \in S_{a\left(\cdot, \tau\left(x_{n}\right)(\cdot), D x_{n}(\cdot)\right)}^{q}, n \geq 1$. From hypothesis $\mathrm{H}(a)$ (iv) we see that $\left\{v_{n}\right\}_{n \geq 1} \subseteq L^{q}\left(Z, \mathbb{R}^{N}\right)$ is bounded and so we may assume that $v_{n} \stackrel{w}{\rightarrow} v$ in $L^{q}\left(Z, \mathbb{R}^{N}\right)$. Then $v_{n}^{*}=-d i v v_{n} \stackrel{w}{\rightarrow}-\operatorname{div} v=v^{*}$ in $W^{-1, q}(Z)$ as $n \rightarrow \infty$.

Let $y \in W_{0}^{1, p}(Z)$ and take $w \in S_{a(\cdot, \tau(x)(\cdot), D y(\cdot))}^{q}$. For each $n \geq 1$ we consider the multifunction $H_{n}: Z \rightarrow 2^{\mathbb{R}^{N}} \backslash\{\emptyset\}$ defined by $H_{n}(z)=\left\{\xi \in a\left(z, \tau\left(x_{n}\right)(z), D y(z)\right)\right.$ : $\left.\|w(z)-\xi\|=d\left(w(z), a\left(z, \tau\left(x_{n}\right)(z), D y(z)\right)\right)\right\}$. We have

$$
G r H_{n}=\operatorname{Gra}\left(\cdot, \tau\left(x_{n}\right)(\cdot), D y(\cdot)\right) \cap\left\{(z, \xi) \in Z \times \mathbb{R}^{N}: \eta_{n}(z, \xi)=0\right\},
$$

where $\eta_{n}(z, \xi)=\|w(z)-\xi\|-d\left(w(z), a\left(z, \tau\left(x_{n}\right)(z), D y(z)\right)\right)$. Evidently $\eta_{n}$ is a Caratheodory function, hence it is jointly measurable. So $G r H_{n} \in \mathcal{L} \times B\left(\mathbb{R}^{N}\right)$ with $\mathcal{L}$ being the Lebesgue $\sigma$-field of $Z$. Using the Yankov-von Neumann-Aumann selection theorem (see Hu-Papageorgiou [7, p. 158) we obtain $w_{n} \in S_{H_{n}(\cdot)}^{q}$. Hence 


$$
\begin{aligned}
& w_{n} \in S_{a\left(\cdot, \tau\left(x_{n}\right)(\cdot), D y(\cdot)\right)}^{q} \text { and } \\
& \qquad w(z)-w_{n}(z) \| \\
& =d\left(w(z), a\left(z, \tau\left(x_{n}\right)(z), D y(z)\right)\right) \\
& \leq h^{*}\left(a(z, \tau(x)(z), D y(z)), a\left(z, \tau\left(x_{n}\right)(z), D y(z)\right)\right)
\end{aligned}
$$

a.e. on $Z$. Since $x_{n} \rightarrow x$ in $L^{p}(Z)$ (Sobolev embedding theorem), we have that $\tau\left(x_{n}\right) \rightarrow \tau(x)$ in $L^{p}(Z)$ and by passing to a subsequence if necessary, we can also say that $\tau\left(x_{n}\right)(z) \rightarrow \tau(x)(z)$ a.e. on $Z$. Because of the lower semicontinuity of $x \rightarrow a(z, x, \xi)$ (see $\mathrm{H}(a)(\mathrm{iii}))$ and proposition I.2.66, p. 61, of Hu-Papageorgiou [7, we have that

$$
h^{*}\left(a(z, \tau(x)(z), D y(z)), a\left(z, \tau\left(x_{n}\right)(z), D y(z)\right)\right) \rightarrow 0 \text { a.e. on } Z .
$$

So $w_{n}(z) \rightarrow w(z)$ a.e. on $Z$ and so from the extended dominated convergence theorem we also have $w_{n} \rightarrow w$ in $L^{q}\left(Z, \mathbb{R}^{N}\right)$. Exploiting the monotonicity of $a\left(z, \tau\left(x_{n}\right)(z), \cdot\right), n \geq 1$, we have

$$
\begin{array}{rl}
0 \leq \int_{Z}\left(v_{n}(z)-w_{n}(z), D x_{n}(z)-D y(z)\right)_{\mathbb{R}^{N}} d z & \\
=\int_{Z}\left(v_{n}(z), D x_{n}(z)-D x(z)\right)_{\mathbb{R}^{N}} & d z+\int_{Z}\left(v_{n}(z), D x(z)-D y(z)\right)_{\mathbb{R}^{N}} d z \\
& +\int_{Z}\left(w_{n}(z), D y(z)-D x_{n}(z)\right)_{\mathbb{R}^{N}} d z \\
=\left\langle v_{n}^{*}, x_{n}-x\right\rangle+\int_{Z}\left(v_{n}(z), D x(z)\right. & -D y(z))_{\mathbb{R}^{N}} d z \\
& +\int_{Z}\left(w_{n}(z), D y(z)-D x_{n}(z)\right)_{\mathbb{R}^{N}} d z .
\end{array}
$$

Recalling that $\limsup _{n \rightarrow \infty}\left\langle v_{n}^{*}, x_{n}-x\right\rangle \leq 0$ and $v_{n} \stackrel{w}{\rightarrow} v, w_{n} \rightarrow w$ in $L^{q}\left(Z, \mathbb{R}^{N}\right)$, we have

$$
\begin{aligned}
0 & \leq \int_{Z}(v(z), D x(z)-D y(z))_{\mathbb{R}^{N}} d z+\int_{Z}(w(z), D y(z)-D x(z))_{\mathbb{R}^{N}} d z \\
& =\langle-\operatorname{div} v-(- \text { divw }), x-y\rangle .
\end{aligned}
$$

But $(y,-$ divw $) \in G r L_{x}$ was arbitrary and from Lemma 1 we know that $L_{x}$ is maximal monotone. So $-\operatorname{div} v \in L_{x}(x)$ and it follows that $v \in S_{a(\cdot, \tau(x)(\cdot), D x(\cdot))}^{q}$.

As before, via a measurable selection argument we obtain $u_{n} \rightarrow v$ in $L^{q}\left(Z, \mathbb{R}^{N}\right)$ such that $u_{n} \in S_{a\left(\cdot, \tau\left(x_{n}\right)(\cdot), D x(\cdot)\right)}^{q}$. Let $u_{n}^{*}=-$ divu $_{n}, n \geq 1, v^{*}=-$ divv. From the choices of $\left\{x_{n}\right\}_{n \geq 1}$ and $\left\{v_{n}^{*}\right\}_{n \geq 1}$ we have

$$
\limsup _{n \rightarrow \infty}\left\langle v_{n}^{*}-v^{*}, x_{n}-x\right\rangle \leq 0,
$$

hence $\lim \sup _{n \rightarrow \infty}\left\langle v_{n}^{*}-u_{n}^{*}, x_{n}-x\right\rangle+\liminf _{n \rightarrow \infty}\left\langle u_{n}^{*}-v^{*}, x_{n}-x\right\rangle \leq 0$. Because $u_{n} \rightarrow v$ in $L^{p}\left(Z, \mathbb{R}^{N}\right)$, we have $u_{n}^{*}=-\operatorname{divu}_{n} \rightarrow-$ divu $=v^{*}$ in $W^{-1, q}(Z)$ and so $\lim \left\langle u_{n}^{*}-v^{*}, x_{n}-x\right\rangle=0$. So we obtain

$$
\limsup _{n \rightarrow \infty}\left\langle v_{n}^{*}-u_{n}^{*}, x_{n}-x\right\rangle \leq 0 .
$$

On the other hand from the monotonicity of $a\left(z, \tau\left(x_{n}\right)(z), \cdot\right)$ (see $\left.\mathrm{H}(a)(\mathrm{ii})\right)$, we have

$$
\liminf _{n \rightarrow \infty}\left\langle v_{n}^{*}-u_{n}^{*}, x_{n}-x\right\rangle \geq 0 .
$$


From (3) and (4) it follows that

$$
\left\langle v_{n}^{*}-u_{n}^{*}, x_{n}-x\right\rangle=\int_{Z}\left(v_{n}(z)-u_{n}(z), D x_{n}(z)-D x(z)\right)_{\mathbb{R}^{N}} d z \rightarrow 0 .
$$

The integrand is nonnegative and so by passing to a subsequence if necessary, we may assume that $\beta_{n}(z)=\left(v_{n}(z)-u_{n}(z), D x_{n}(z)-D x(z)\right)_{\mathbb{R}^{N}} \rightarrow 0$ a.e. on $Z$ and $\left|\beta_{n}(z)\right| \leq k_{1}(z)$ a.e. on $Z$ for all $n \geq 1$, with $k_{1} \in L^{1}(Z)$. From hypotheses $\mathrm{H}(a)$ (iv) and (v), for all $z \in Z \backslash N,|N|=0(|\cdot|$ being the Lebesgue measure on $Z$ ), we have

$$
\begin{aligned}
k_{1}(z) \geq & \left(v_{n}(z)-u_{n}(z), D x_{n}(z)-D x(z)\right)_{\mathbb{R}^{N}} \\
\geq & \gamma_{1}\left(\left\|D x_{n}(z)\right\|^{p}+\|D x(z)\|^{p}\right)-2 \gamma_{2} \\
& -\left\|D x_{n}(z)\right\|\left(b_{1}(z)+c_{1}\left|\tau\left(x_{n}\right)(z)\right|^{p-1}+c_{1}\|D x(z)\|^{p-1}\right) \\
& -\|D x(z)\|\left(b_{1}(z)+c_{1}\left|\tau\left(x_{n}\right)(z)\right|^{p-1}+c_{1}\left\|D x_{n}(z)\right\|^{p-1}\right) .
\end{aligned}
$$

From (5) it follows that for all $z \in Z \backslash N,\left\{D x_{n}(z)\right\}_{n \geq 1} \subseteq \mathbb{R}^{N}$ is bounded and so by passing to a subsequence (depending in general on $z \in Z \backslash N$ ) if necessary, we may assume that $D x_{n}(z) \rightarrow \hat{\xi}(z), z \in Z \backslash N$. We can find $g_{n}(z) \in a(z, \tau(x)(z), \hat{\xi}(z))$ such that

$$
\begin{aligned}
\left\|v_{n}(z)-g_{n}(z)\right\| & =d\left(v_{n}(z), a(z, \tau(x)(z), \hat{\xi}(z))\right) \\
& \leq h^{*}\left(a\left(z, \tau\left(z_{n}\right)(z), D x_{n}(z)\right), a(z, \tau(x)(z), \hat{\xi}(z))\right) .
\end{aligned}
$$

Let $s_{n}(z) \in a\left(z, \tau\left(x_{n}\right)(z), D x_{n}(z)\right)$ such that

$$
d\left(s_{n}(z), a(z, \tau(x)(z), \hat{\xi}(z))\right)=h^{*}\left(a\left(z, \tau\left(z_{n}\right)(z), D x_{n}(z)\right), a(z, \tau(x)(z), \hat{\xi}(z))\right) .
$$

The sequence $\left\{s_{n}(z)\right\}_{n \geq 1} \subseteq \mathbb{R}^{N}$ is bounded and so we may assume that $s_{n}(z) \rightarrow$ $s(z)$ in $\mathbb{R}^{N}$. Because $\left(\tau\left(x_{n}\right)(z), D x_{n}(z), s_{n}(z)\right) \in \operatorname{Gra}(z, \cdot, \cdot), n \geq 1$, we obtain $(\tau(x)(z), \hat{\xi}(z), s(z)) \in \operatorname{Gra}(z, \cdot, \cdot)$ (see $\mathrm{H}(a)(\mathrm{iii}))$. Therefore

$$
h^{*}\left(a\left(z, \tau\left(z_{n}\right)(z), D x_{n}(z)\right), a(z, \tau(x)(z), \hat{\xi}(z))\right) \rightarrow 0
$$

and so $\left\|v_{n}(z)-g_{n}(z)\right\| \rightarrow 0$. Note that $\left\{g_{n}\right\}_{n \geq 1} \subseteq a(z, \tau(x)(z), \hat{\xi}(z))$ and so we may assume that $g_{n}(z) \rightarrow \hat{g}(z) \in a(z, \tau(z)(z), \hat{\xi}(z))$. Hence finally $v_{n}(z) \rightarrow$ $\hat{g}(z) \in a(z, \tau(x)(z), \hat{\xi}(z))$ for all $z \in Z \backslash N$. Recall that for $z \in Z \backslash N$ we have $\left(v_{n}(z)-u_{n}(z), D x_{n}(z)-D x(z)\right)_{\mathbb{R}^{N}} \rightarrow 0$. So in the limit we have $(\hat{g}(z)-v(z)$, $\hat{\xi}(z)-D x(z))_{\mathbb{R}^{N}}=0$ and because $a(z, \tau(x)(z), \cdot)$ is strictly monotone (see $\left.\mathrm{H}(a)(\mathrm{ii})\right)$, it follows that $\hat{\xi}(z)=D x(z)$ for all $z \in Z \backslash N$. Thus we have that $D x_{n}(z) \rightarrow D x(z)$ for all $z \in Z \backslash N$. Also from hypotheses $\mathrm{H}(a)$ (iv) and (v) we can easily check that $\left\{\left\|D x_{n}(\cdot)\right\|^{p}\right\}_{n \geq 1}$ is uniformly integrable. Because $D x_{n} \stackrel{w}{\rightarrow} D x$ in $L^{p}\left(Z, \mathbb{R}^{N}\right)$, from Vitali's theorem we have that $D x_{n} \rightarrow D x$ in $L^{p}\left(Z, \mathbb{R}^{N}\right)$ and so we conclude that $x_{n} \rightarrow x$ in $W_{0}^{1, p}(Z)$. Therefore $V_{1}$ is of type $S_{+}$.

Next let $N: W_{0}^{1, p}(Z) \rightarrow L^{q}(Z)$ and $B: L^{p}(Z) \rightarrow L^{q}(Z)$ be defined by $N(x)(\cdot)=$ $f(\cdot, \tau(x)(\cdot), D \tau(x)(\cdot))$ and $B x(\cdot)=\beta(\cdot, x(\cdot))$. Also let $G: W_{0}^{1, p}(Z) \rightarrow P_{f c}\left(L^{r^{\prime}}(Z)\right)$ be defined by $G(x)=S_{E(\cdot, x(\cdot))}^{r^{\prime}}$. Since $L^{q}(Z)$ and $L^{r^{\prime}}(Z)$ are embedded continuously in $W^{-1, q}(Z)$ we can define $U: W_{0}^{1, p}(Z) \rightarrow P_{w k c}\left(W^{-1, q}(Z)\right)$ by $U(x)=V_{1}(x)+$ $N(x)+M \tau(x)+\rho B(x)+G(x)$.

Proposition 3. If hypotheses $\mathrm{H}(a), \mathrm{H}_{0}, \mathrm{H}(j), \mathrm{H}(f), \mathrm{H}(\theta)$ hold, then $U$ is pseudomonotone and for $\rho>0$ large it is coercive. 
Proof. Suppose $x_{n} \stackrel{w}{\rightarrow} x$ in $W_{0}^{1, p}(Z), x_{n}^{*} \stackrel{w}{\rightarrow} x^{*}$ in $W^{-1, q}(Z), x_{n}^{*} \in U\left(x_{n}\right), n \geq 1$, and $\lim \sup _{n \rightarrow \infty}\left\langle x_{n}^{*}, x_{n}-x\right\rangle \leq 0$. Because $U$ is bounded, it suffices to show that $x^{*} \in V_{1}(x)$ and $\left\langle x_{n}^{*}, x_{n}\right\rangle \rightarrow\left\langle x^{*}, x\right\rangle$ (see Hu-Papageorgiou [7], proposition III.6.11, p. 366). We have $x_{n}^{*}=v_{n}^{*}+N\left(x_{n}\right)+M \tau\left(x_{n}\right)+\rho B\left(x_{n}\right)+g_{n}$, with $v_{n}^{*} \in V_{1}\left(x_{n}\right), g_{n} \in$ $G_{1}\left(x_{n}\right)$. Because of hypotheses $\mathrm{H}(j)(\mathrm{iii}),\left\{g_{n}\right\}_{n \geq 1} \subseteq L^{r^{\prime}}(Z)$ is bounded and so we may assume that $g_{n} \stackrel{w}{\rightarrow} g$ in $L^{r^{\prime}}(Z)$. Note that $x_{n} \rightarrow x, \tau\left(x_{n}\right) \rightarrow \tau(x)$ in $L^{p}(Z)$ and by passing to a subsequence if necessary, we may also assume that $x_{n}(z) \rightarrow x(z), \tau\left(x_{n}\right)(z) \rightarrow \tau(x)(z)$ a.e. on $Z$. Using the closedness of the graph of $\partial j(z, \cdot)$ and of $Q(z, \cdot)$ and proposition VII.3.9, p. 694, of Hu-Papageorgiou [7], we have that

$$
\begin{aligned}
g(z) & \in \operatorname{conv} \limsup _{n \rightarrow \infty}\left[\partial j\left(z, \tau\left(x_{n}\right)(z)\right) \cap Q\left(z, x_{n}(z)\right)\right] \\
& \subseteq \operatorname{conv}\left[\limsup _{n \rightarrow \infty} \partial j\left(z, \tau\left(x_{n}\right)(z)\right) \cap \limsup _{n \rightarrow \infty} Q\left(z, x_{n}(z)\right)\right] \\
& \subseteq \operatorname{conv}[\partial j(z, \tau(x)(z)) \cap Q(z, x(z))] \text { a.e. on } Z \text {, i.e. } g \in G(x) .
\end{aligned}
$$

Moreover, because $1 \leq r<p^{*}, W_{0}^{1, p}(Z)$ is embedded compactly in $L^{r}(Z)$ and so $x_{n} \rightarrow x$ in $L^{r}(Z)$. Hence $\left\langle g_{n}, x_{n}-x\right\rangle=\int_{Z} g_{n}(z)\left(x_{n}(z)-x(z)\right) d z \rightarrow 0$. Similarly we have $\left\langle B\left(x_{n}\right), x_{n}-x\right\rangle \rightarrow 0$ and $\left\langle N\left(x_{n}\right), x_{n}-x\right\rangle \rightarrow 0$. So finally we obtain that $\lim \sup _{n \rightarrow \infty}\left\langle v_{n}^{*}, x_{n}-x\right\rangle \leq 0$. But from Proposition 2 we know that $V_{1}$ is of type $S_{+}$. So $x_{n} \rightarrow x$ in $W_{0}^{1, p}(Z)$. Therefore $N\left(x_{n}\right) \rightarrow N(x)$ in $L^{q}(Z)$, hence in $W^{-1, q}(Z)$, too. Also $B\left(x_{n}\right) \rightarrow B(x)$ in $W^{-1, q}(Z)$. We have $v_{n}^{*}=-\operatorname{div}_{n}, v_{n} \in S_{a\left(\cdot, \tau\left(x_{n}\right)(\cdot), D x_{n}(\cdot)\right)}^{q}$ and $v_{n} \stackrel{w}{\rightarrow} v$ in $L^{q}\left(Z, \mathbb{R}^{N}\right)$, hence $v_{n}^{*}=-\operatorname{div}_{n} \stackrel{w}{\rightarrow}-\operatorname{div} v=v^{*}$ in $W^{-1, q}(Z)$. Because $\tau\left(x_{n}\right) \rightarrow \tau(x)$ in $W_{0}^{1, p}(Z)$ and $\operatorname{Gra}(z, \cdot, \cdot)$ is closed (see $\mathrm{H}(a)($ iii)), as above we obtain $v(z) \in$ conv $\lim \sup a\left(z, \tau\left(x_{n}\right)(z), D x_{n}(z)\right) \subseteq a(z, \tau(x)(z), D x(z))$ a.e. on $Z$, hence $v^{*} \in V_{1}(x)$. Thus we have $x^{*}=v^{*}+N(x)+M \tau(x)+\rho B(x)+g$, with $v^{*} \in V_{1}(x), g \in G(x)$, i.e. $x^{*} \in U(x)$. Also it is easy to see that $\left\langle x_{n}^{*}, x_{n}\right\rangle \rightarrow\left\langle x^{*}, x\right\rangle$. This proves the pseudomonotonicity of $U$.

For every $x \in W_{0}^{1, p}(Z)$ and every $x^{*} \in U(x)$ we have $\left\langle x^{*}, x\right\rangle=\left\langle v^{*}, x\right\rangle+$ $\langle N(x), x\rangle+\langle M \tau(x), x\rangle+\rho\langle B(x), x\rangle+\langle g, x\rangle$. From hypothesis $\mathrm{H}(a)(\mathrm{v})$ we have $\left\langle v^{*}, x\right\rangle \geq \gamma_{1}\|D x\|_{p}^{p}-\left\|\gamma_{2}\right\|_{1}$. Also from hypothesis $\mathrm{H}(f)$ (iii) we have $\langle N(x), x\rangle \geq$ $-c_{7}\|x\|_{1, p}^{p-1}\|x\|_{p}-c_{8}\|x\|_{p}-c_{9}, c_{7}, c_{8}, c_{9}>0$. Using Young's inequality with $\varepsilon>0$ we obtain $\|x\|_{1, p}^{p-1}\|x\|_{p} \leq \frac{1}{\varepsilon^{p} p}\|x\|_{p}^{p}+\frac{\varepsilon^{q}}{q}\|x\|_{1, p}^{p}$ (recall $p-1=\frac{p}{q}$ ) and so $\langle N(x), x\rangle \geq$ $-c_{7} \frac{1}{\varepsilon^{p} p}\|x\|_{p}^{p}-c_{7} \frac{\varepsilon^{q}}{q}\|x\|_{1, p}^{p}-c_{8}\|x\|_{p}-c_{9}$. From the properties of the penalty function, we have

$$
\rho\langle B(x), x\rangle \geq c_{5} \rho\|D x\|_{p}^{p}-c_{6} \rho .
$$

Also from hypothesis $\mathrm{H}(j)$ (iii) and the compact embedding of $W_{0}^{1, p}(Z)$ in $L^{r}(Z)$ we have that $\langle g, x\rangle \geq-c_{10}\|x\|_{1, p}, c_{10}>0$. So finally via Poincaré's inequality we obtain

$$
\left\langle x^{*}, x\right\rangle \geq\left(c_{11}-\frac{c_{7} \varepsilon^{q}}{q}\right)\|x\|_{1, p}^{p}+\left(\rho c_{5}-\frac{c_{7}}{\varepsilon^{p} p}\right)\|x\|_{p}^{p}-c_{10}\|x\|_{1, p}-c_{12}(\rho)
$$

with $c_{11}, c_{12}(\rho)>0$.

First choose $\varepsilon>0$ so that $c_{11}>\frac{c_{7} \varepsilon^{q}}{q}$ and then with this choice of $\varepsilon>0$ choose $\rho>0$ large enough to have $\rho c_{5}>\frac{c_{7}}{\varepsilon^{p} p}$. With these choices, from (6) we infer that $U$ is coercive. 
Using this proposition we can have the following existence result for the auxiliary problem (2):

Proposition 4. If hypotheses $\mathrm{H}(a), \mathrm{H}_{0}, \mathrm{H}(j), \mathrm{H}(f)$ and $\mathrm{H}(\theta)$ hold, then for $\rho>0$ large enough, problem (2) has a solution $x \in W_{0}^{1, p}(Z)$.

Proof. From Proposition 3 we know that for large $\rho>0$, the operator $U$ is pseudomonotone, coercive, hence it is surjective (see Hu-Papageorgiou [7], p. 372). So we can find $x \in W_{0}^{1, p}(Z)$ such that $U(x)=\theta(w)+M w$. Evidently this is a solution of $(2)$.

\section{EXISTENCE THEOREM}

Our existence theorem for problem (1) will be based on the following fixed point result of Heikkila-Hu [5]:

Theorem 5 ([5]).

If $X$ is a separable reflexive ordered Banach space, $K \subseteq X$ is nonempty and weakly closed and $S: K \rightarrow 2^{K} \backslash\{\emptyset\}$ is a multifunction with weakly closed values such that (i) the set $M=\{x \in K: x \leq y$ for some $y \in S(x)\}$ is nonempty and (ii) $x_{1} \leq y_{1} \in S\left(x_{1}\right)$ and $x_{1} \leq x_{2}$ imply that for some $y_{2} \in S\left(x_{2}\right)$ we have $y_{1} \leq y_{2}$,

then $S$ has a fixed point in $K$.

Using this fixed point theorem we can prove the following existence result for problem (1).

Theorem 6. If hypotheses $\mathrm{H}(a), \mathrm{H}_{0}, \mathrm{H}(j), \mathrm{H}(f)$ and $\mathrm{H}(\theta)$ hold, then problem (1) has a solution $x \in W_{0}^{1, p}(Z)$.

Proof. Let $K \subseteq W_{0}^{1, p}(Z)$ be the order interval $[\psi, \phi]=\left\{x \in W^{1, p}(Z): \psi(z) \leq\right.$ $x(z) \leq \phi(z)$ a.e. on $Z\}$. Evidently $K$ is weakly closed. We consider the multifunction $S: K \rightarrow 2^{W_{0}^{1, p}(Z)} \backslash\{\emptyset\}$ which to each $w \in K$ assigns the set of solutions of the auxiliary problem (2). From Proposition 4 we know that for all $w \in K, S(w) \neq \emptyset$ and it is clear from the proof of Proposition 3 that for all $w \in K, S(w) \subseteq W^{1, p}(Z)$ is weakly closed.

Claim I: $S(K) \subseteq K$.

Let $x \in S(w), w \in K$. We have

$$
v^{*}+N(x)+M \tau(x)+\rho B(x)+g=\theta(w)+M w
$$

with $v^{*} \in V_{1}(x), v^{*}=-\operatorname{div} v, v \in S_{a(\cdot, \tau(x)(\cdot), D x(\cdot))}^{q}, g \in G(x)$.

Since $\psi \in W^{1, p}(Z)$ is a lower solution, using as a test function $(\psi-x)^{+} \in$ $W_{0}^{1, p}(Z)$ (recall $\psi_{\left.\right|_{\Gamma}} \leq 0$ ), we have

$$
\begin{aligned}
\int_{Z}\left(v_{-}(z), D(\psi-x)^{+}(z)\right)_{\mathbb{R}^{N}} d z+\int_{Z} f(z, \psi(z), D \psi(z))(\psi-x)^{+}(z) d z \\
+\int_{Z} u_{-}(z)(\psi-x)^{+}(z) d z \leq \int_{Z} \theta(\psi)(z)(\psi-x)^{+}(z) d z
\end{aligned}
$$


Also from (7) we have

$$
\begin{gathered}
\int_{Z}\left(v(z), D(\psi-x)^{+}(z)\right)_{\mathbb{R}^{N}} d z+\int_{Z} f(z, \tau(x)(z), D \tau(x)(z))(\psi-x)^{+}(z) d z \\
+\rho \int_{Z} \beta(z, x(z))(\psi-x)^{+}(z) d z+\int_{Z} g(z)(\psi-x)^{+}(z) d z \\
=\int_{Z} \theta(w(z))(\psi-x)^{+}(z) d z+\int_{Z} M(w(z)-\tau(x)(z))(\psi-x)^{+}(z) d z
\end{gathered}
$$

Subtracting (9) from (8) and using the definitions of $\tau, \beta, G$ and the monotonicity of $a(z, \psi(z), \cdot)$, we obtain

$$
\begin{aligned}
& -\rho \int_{Z} \beta(z, x(z))(\psi-x)^{+}(z) d z \\
\leq & \int_{\{\psi>x\}}(-\theta(\psi(z))+M \psi(z)-\theta(w(z))-M w(z))(\psi-x)(z) d z \leq 0
\end{aligned}
$$

hence $-\int_{\{\psi>x\}}\left(|x(z)|^{p-2} x(z)-|\psi(z)|^{p-2} \psi(z)\right)(\psi(z)-x(z)) d z \leq 0$, a contradiction unless $\psi(z) \leq x(z)$ a.e. on $Z$. Similarly we can show that $x(z) \leq \phi(z)$ a.e. on $Z$. This proves Claim I.

Claim II: If $w_{1} \in K, w_{1} \leq x_{1} \in S\left(w_{1}\right)$ and $w_{1} \leq w_{2}$, then we can find $x_{2} \in S\left(w_{2}\right)$ such that $x_{1} \leq x_{2}$ (on $W^{1, p}(Z)$ we consider the partial order induced by the positive cone $\left.L^{p}(Z)_{+}\right)$.

Since $x_{1} \in S\left(w_{1}\right) \subseteq K$ (Claim I), we have $B\left(x_{1}\right)=0, \tau\left(x_{1}\right)=x_{1}, D \tau\left(x_{1}\right)=D x_{1}$ and $Q\left(z, x_{1}(z)\right)=\mathbb{R}$. So we have

$$
v_{1}^{*}+N\left(x_{1}\right)+M x_{1}+g=\theta\left(w_{1}\right)+M w_{1},
$$

with $v_{1}^{*}=-\operatorname{divv}_{1}, v_{1} \in L_{a\left(\cdot, x_{1}(\cdot), D x_{1}(\cdot)\right)}^{q}, N\left(x_{1}\right)(\cdot)=f\left(\cdot, x_{1}(\cdot), D x_{1}(\cdot)\right)$ and $g \in$ $S_{\partial j(\cdot, x(\cdot))}^{r^{\prime}}$. Because $(\theta+M)(\cdot)$ is nondecreasing (see $\left.\mathrm{H}(\theta)\right)$, we have $\theta\left(w_{1}(z)\right)+$ $M w_{1}(z) \leq \theta\left(w_{2}(z)\right)+M w_{2}(z)$ a.e. on $Z$. So for all $y \in W_{0}^{1, p}(Z)_{+}$(i.e. $y \in$ $W_{0}^{1, p}(Z), y(z) \geq 0$ a.e. on $Z$ ) we have

$$
\begin{aligned}
\left\langle v_{1}^{*}, y\right\rangle+ & \int_{Z} f\left(z, x_{1}(z), D x_{1}(z)\right) y(z) d z+M \int_{Z} x_{1}(z) y(z) d z \\
& +\int_{Z} g(z) y(z) d z \leq \int_{Z}\left(\theta\left(w_{2}(z)\right)+M w_{2}(z)\right) y(z) d z,
\end{aligned}
$$

which implies that $x_{1} \in W_{0}^{1, p}(Z)$ is a lower solution of the boundary value problem

$$
\left\{\begin{array}{l}
-\operatorname{diva}(z, x(z), D x(z))+f(z, x(z), D x(z))+M x(z)+\partial j(z, x(z)) \\
\ni \theta\left(w_{2}(z)\right)+M w_{2}(z) \text { a.e. on } Z, \\
x_{\left.\right|_{\Gamma}}=0 .
\end{array}\right\}
$$

Note that $\phi \in W^{1, p}(Z)$ is an upper solution of (10), too (see $\mathrm{H}(\theta)$ ). So as in the first part of the proof we can obtain a solution $x_{2} \in W_{0}^{1, p}(Z)$ of $(10)$ such that $x_{1}(z) \leq x_{2}(z) \leq \phi(z)$ a.e. on $Z$. Therefore $x_{2} \in S\left(w_{2}\right)$ and $x_{1} \leq x_{2}$. This proves the claim.

Claims I and II permit the use of Theorem 5 with $X=W^{1, p}(Z), K=[\phi, \psi]$ and $S$ as above. So we obtain $x \in W_{0}^{1, p}(Z) \cap K$ such that $x \in S(x)$. Clearly this $x$ is a solution of problem (1). 
Remark. As a continuation of this study, one could look for conditions which will guarantee the existence of a greatest and of a least solution (extremal solutions) in $K=[\psi, \phi]$ (see Stuart [16]).

\section{ACKNOWLEDGEMENT}

The authors thank the referee for his constructive comments and remarks.

\section{REFERENCES}

1. Ambrosetti, A - Badiale, M.: "The dual variational principle and elliptic problems with discontinuous nonlinearities" J. Math. Anal. Appl. 140 (1989), 363-373. MR 90k:35097

2. Carl, S. - Dietrich, H.: "The weak upper and lower solution method for quasilinear elliptic equations with generalized subdifferentiable perturbations" Applic. Anal. 56 (1995), 263-278. MR 97f:35045

3. Chang, K. C.: "Variational methods for nondifferentiable functionals and their applications to partial differential equations" J. Math. Anal. Appl. 80 (1981), 102-129. MR 82h:35025]

4. Deuel, J. - Hess, P.: "A criterion for the existence of solutions for nonlinear elliptic boundary value problems" Proc. Roy. Soc. Edinburgh Sect. A 74 (1974/75), 49-54. MR 55:13070

5. Heikkila, S. - Hu, S.: "On fixed points of multifunctions in ordered spaces" Applic. Anal. 51 (1993), 115-127. MR 95a:54062

6. Heikkila, S. - Lakshmikantham, V.: "Monotone Iterative Techniques for Discontinuous Nonlinear Differential Equations" Marcel-Dekker, New York (1994). MR 95d:34002

7. Hu, S. - Papageorgiou, N. S.: "Handbook of Multivalued Analysis. Volume I: Theory" Kluwer, Dordrecht, The Netherlands (1997). MR 98k:47001

8. Hu, S. - Papageorgiou, N. S.: "Handbook of Multivalued Analysis. Volume II: Applications" Kluwer, Dordrecht, The Netherlands (2000). MR 2001g:49001

9. Kourogenis, N. C. - Papageorgiou, N. S.: "Nonsmooth critical point theory and nonlinear elliptic equations at resonance" J. Austral. Math. Soc. (Series A) 69 (2000), 245-271. MR 2001m:35078

10. Le, V. K.: "On some equivalent properties of sub- and supersolutions in second order quasilinear elliptic equations" Hiroshima Math. Jour. 28 (1998), 373-380. MR 99h:35049

11. Le, V. K.: "Subsolution-supersolution method in variational inequalities" Nonlin. Anal. 45 (2001), 775-800. MR 2002c:35126

12. Naniewicz, Z. - Panagiotopoulos, P.: "Mathematical Theory of Hemivariational Inequalities and Applications" Marcel-Dekker, New York (1995). MR 96d:47067

13. Panagiotopoulos, P.: "Hemivariational Inequalities: Applications to Mechanics and Engineering" Springer-Verlag, New York (1993).

14. Papageorgiou, N. S. - Papalini, F. - Vercillo, S.: "Minimal solutions for nonlinear parabolic problems with unilateral constraints" Houston J. Math. 23 (1997), 189-201. MR 2000g:35124

15. Showalter, R.: "Monotone Operators in Banach Space and Nonlinear Partial Differential Equations" Math. Surveys and Monographs, Vol. 49, AMS, Providence, R.I. (1997). MR 98c: 47076

16. Stuart, C.: "Maximal and minimal solutions of elliptic differential equations with discontinuous nonlinearities" Math Z. 163 (1978), 239-249. MR 80g:35059

Department of Mathematics, National Technical University, Zografou Campus, Athens 157 80, Greece

Current address: Department of Financial Management and Banking, University of Pireus, Pireus, Greece

Department of Mathematics, National Technical University, Zografou Campus, Athens 157 80, Greece

E-mail address: npapg@math.ntua.gr 